\title{
CULTURA POLÍTICA PARTICIPATIVA Y ACTORES EMERGENTES: APROXIMACIONES DESDE LA EXPERIENCIA VENEZOLANA (1999-2014).
}

PARTICIPATIVE POLITICAL CULTURE AND EMERGING ACTORS: APPROACHES FROM THE VENEZUELAN EXPERIENCE (1999-2014).

\author{
Mila Ivanovic* \\ Enrique Rey Torres**
}

RESUMEN: Durante los últimos años, el campo político de la organización popular en Venezuela ha experimentado la emergencia de nuevos actores sociales que vienen a disputar y generar tensiones en el ámbito de la participación popular. La complejidad de estos procesos en curso, se encuentra minada de múltiples lecturas que tienden, por un lado, a romantizar a los actores que se movilizan en los territorios populares, y por el otro, a restarle legitimidad e importancia política a sus prácticas. Este artículo busca rastrear las tensiones existentes en el proceso político de la participación en Venezuela. Basado en una investigación en curso en varios sectores de Caracas, se buscará poner de manifiesto bajo qué modalidad, los consejos comunales, y la etapa siguiente constituida alrededor de las Comunas, han amalgamado una serie de actores inesperados y a veces "indeseables" ("colectivos", jóvenes sin afiliación política y sindicatos de la construcción) que tensionan y atraviesan las prácticas sociales desplegadas en la participación. En este contexto, se tratará de responder a algunas interrogantes acerca de la naturaleza y la potencialidad de las Comunas. En particular, al analizar que tipo de ruptura y/o reproducción se generan en las prácticas de estos actores.

Palabras claves: politización; cultura política; territorialidad; democracia participativa.

\footnotetext{
* Doctora en Ciencia Política, Investigadora-docente del Instituto de Altos Estudios Nacionales (IAEN), Quito, Ecuador; investigadora del Centro de Estudios Latinoamericanos Rómulo Gallegos (CELARG), Caracas, Venezuela; miembro del Grupo Venezolano de Investigación sobre Territorios populares; E-mail: milaivanovic@hotmail.com

** Maestría en Sociología, Profesor en la Universidad Central de Venezuela(UCV), Caracas, Venezuela; Investigador del Centro de Estudios Latinoamericanos Rómulo Gallegos (CELARG), Caracas, Venezuela; y miembro del Grupo Venezolano de investigación sobre Territorios Populares; E-mail: enriquert82@gmail.com
} 
ABSTRACT: In recent years, the political camp of popular organization in Venezuela has seen the emergence of new social actors who have created tensions and contestations in the realm of popular participation. The complexity of these processes have given rise to multiple analyses which tend, on the one hand, to romanticize the actors who mobilize in popular territories, and on the other, to delegitimize their practices and political importance. This article seeks to trace the tensions in the political process of participation in Venezuela. Based on ongoing fieldwork in various sectors of Caracas, we seek to show how Community Councils, and their offshoots , "Communes", have brought together a series of unexpected, and sometimes 'undesirable' actors (the so-called 'colectivos', groups of young people without political affiliations and construction unions) that antagonise and cut through the social practices deployed by participation. In this context, we address questions about the nature and potential of the Communes. In particular, we analyse the different types of rupture and/or reproduction that are generated by.

Keywords: politicization; political culture; territoriality; participatory democracy.

\section{INTRODUCCIÓN}

Desde 1999, los actores y las subjetividades políticas que confluyen en el contexto venezolano se han reconfigurado. La primera elección presidencial que llevó al poder a Hugo Chávez, puso fin a 40 años de cohabitación "pactada" entre dos partidos políticos: Acción Democrática y COPEI (el primero es miembro de la Internacional Socialista y el segundo es de tendencia demócrata cristiana) ${ }^{1}$. En este contexto, las élites políticas y económicas se van renovando, en la misma medida que el modelo político comienza a experimentar cambios sustanciales en su arquitectura. Inmediatamente después de las elecciones presidenciales de 1998, los/as electores/as venezola-

\footnotetext{
${ }^{1}$ Con el ascenso electoral de Hugo Chávez y la revolución bolivariana, los procesos de maximización del consenso y minimización de conflicto entre las principales élites políticas del país (centrales sindicales, iglesia, cámaras empresariales y partidos políticos) se diluyeron. A su vez, en un primer momento, los partidos políticos, en tanto modalidad de articulación y artífices del consenso, perdieron su eficacia simbólica.
} 
nos/as adoptan una nueva Constitución que consagra la "democracia participativa y protagónica" como modelo político de la República Bolivariana. La noción de democracia protagónica es central en el discurso bolivariano y en la Constitución de 1999. Se sitúa en la frontera entre la democracia directa y la democracia participativa, y busca dar un papel de protagonistas políticos activos e informados a los/as ciudadanos/as, apoyándose en las posibilidades de referendos revocatorios de los dirigentes combinados con diversas formas de participación en la base. A pesar de estar avalado por una refundación republicana donde prevalecen los ideales del Libertador Simón Bolívar, el primer periodo de gobierno, es un momento de estancamiento en materia de avances participativos. Si bien la Constituyente representó una interesante fuente de innovación en la materia, en este aspecto los resultados distaron de ser significativos ${ }^{2}$.

Es a partir del 2003-2004 que se inicia el giro "participativista”, que más allá de estrategias previas de movilización y organización, se vuelve una política de Estado, luego de un periodo de fuerte conflictividad política (golpe de Estado fallido, lock-out patronal, referendo revocatorio). El ritmo de creación de las instituciones participativas se acelera. Con los primeros dispositivos, que son los Consejos Locales de Planificación Pública (CLPP), los actores institucionales y populares comienzan a trabajar, y la participación local comienza a adquirir contornos más precisos. Sin embargo, en las filas del chavismo, la iniciativa no prende ${ }^{3}$.

A fines de 2005, y a través de la aprobación de la Ley de Consejos Comunales (CC), la participación se vuelve a dinamizar; se extiende a través de todo el país articulando, de esta forma, a organizaciones como los Círculos Bolivarianos, los Comités de Tierras Urbanas, de Salud y las Unidades de Batalla Endógena. Con más de 40 mil consejos registrados, estas organizaciones se han convertido en

\footnotetext{
${ }^{2}$ Aunque no es intención de los/as autores/as realizar una evaluación de éste período, es necesario destacar que en parte, el estancamiento que en materia participativa se experimentó durante ese período, pudo estar relacionado, por un lado, a la dinámica conflictiva posterior a la ruptura definitiva con los esquemas de negociación y definición de consensos del pacto de Puntofijo.

${ }^{3}$ Lo que no sucedió en los sectores de oposición, que encontraron una herramienta de mediación política entre los grupos sociales y las instituciones locales en manos de los partidos de oposición. Podemos considerar que estas organizaciones fueron las primeras iniciativas en organizar institucionalmente "desde arriba" (y fueron apropiadas "desde abajo") a los diferentes sectores de la sociedad.
} 
la piedra de toque de toda la arquitectura participativa del país. ¿Podemos por eso afirmar que los CC son la expresión de la emergencia del "poder popular"? ¿Es acertado considerarlos como modelos de participación a partir de los cuales podemos replantearnos un renacer democrático? ¿Cómo se articula en última instancia los cambios políticos ocurridos y las dinámicas de organización territorial, reflejos de resistencia a la dominación ejercida desde la función empeñada por clases populares periféricas, vivero de mano de obra barata, en un entorno laboral desregulado y sujeto a un anclaje informal?

Con base en una investigación actual ${ }^{4}$, veremos en qué medida, estas organizaciones, y la etapa siguiente constituida alrededor de las Comunas han amalgamado a una serie de actores inesperados, y a veces "indeseables" ("colectivos", jóvenes desafiliados y sindicatos de la construcción) que dan muestra del dinamismo de la democracia participativa venezolana. Por ello, creemos indispensable formular desde una experiencia etnográfica, hipótesis que asimilan trabajo previo alrededor de la cultura política adquirida en en el tránsito a una democracia participativa, y mutaciones tanto de los actores como de la ideología que acompaña la construcción de otros patrones políticos. Si bien se ha dicho bastante sobre el proceso venezolano respecto de la participación, el poder popular o el sistema político del nuevo régimen democrático (García Guadilla, 2008; López Maya 2008; Vargas, 2010, Addor 2012, Ivanovic 2013), poca atención se le ha dado a la transformación o evoluciones de este proceso político, y menos a actores "marginales" del chavismo. Para distanciarnos de posturas idealistas y propensas a la inmediatez, y anclarnos en el "tiempo mediano" de la participación, presentaremos resultados provisorios de un estudio llevado a cabo simultáneamente en Caracas en dos barrios populares (Santa Rosalía y Antímano) con

\footnotetext{
${ }^{4}$ Esta investigación contempla el uso de métodos cualitativos y trabajo intensivo de campo (etnografia política). Los resultados preliminares que se presentan en este trabajo obedecen a los hallazgos obtenidos en dos de los estudios de caso que contempla la investigación. El muestreo de selección de ambos casos fue intencionado y no aleatorio. Los criterios considerados para la selección fueron a) territorios populares y; b) niveles variables de formalización y consolidación de la organización local. Dentro del equipo del Grupo Venezolano de Investigación sobre Territorios Populares, caracterizamos a estos últimos como: asentamientos humanos con grados significativos de exclusión tanto física (al margen de la trama urbana, con déficit en servicios e infraestructura, etc.) social (pobreza, desempleo, etc.) y económica (ausencia de fuentes de empleo y de actividades productivas insertas en la economía formal).
} 
niveles de organización disímiles 5 .

En cuanto al marco conceptual, existen numerosos trabajos sobre la democratización y la politización de los sectores populares en América Latina (Auyero, 2001; Holston, 2007; Merklen, 2005; Ciccarielo-Maher, 2013), sin embargo pocos consideran el tiempo medio de los acontecimientos políticos, dando a entender que tipo de relaciones se tejen entre los actores, receptores de políticas de corte participacionistas, sus niveles de incidencia y los cambios de modelos políticos. Por ello, creemos necesario volcarnos hacia la territorialidad en cuanto modalidad de resistencia de las clases populares a la dominación (Rosa Bonheur, 2014).

Organizaremos nuestra argumentación en cinco partes: la historia de la participación en Venezuela, las prácticas concretas de los Consejos Comunales, las perspectivas de ampliación de la participación con sus horizontes e impasses, el surgimiento de una cultura política participativa y su extensión a actores inesperados.

\section{LAS PRIMERAS EXPERIENCIAS DE PARTICIPACIÓN EN VENEZUELA: ENTRE MODERNIZACIÓN ESTATAL Y REVOLUCIÓN}

En un clima de múltiples tensiones sociales, el inicio de la década de los 90 está signado por la tentativa de reforma política del sistema político de conciliación de élites ${ }^{6}$ que se encuentra entonces en su fase final. Las posibilidades formales de la participación como nuevo instrumento de un discurso tecnocrático y modernizador aparecen entonces a través del proceso de descentralización de los años setenta y ochenta, y como respuesta a la ineficiencia de la gestión centralizada de los servicios públicos locales. Hasta 1979, las elecciones de los diferentes puestos políticos se realizan de manera simultánea, lo que favorece la homogeneidad política de los gobernantes.

\footnotetext{
${ }_{5}^{5}$ Los dos constituyeron su Comuna, la primera en un grado de gran conflictividad interna a la comunidad, la otra con mayor cohesión y respaldo institucional.

${ }^{6}$ Para Juan Carlos Rey (1991) el sistema político de conciliación de élites, constituido en la post-dictadura perezjimenista, dio lugar a la creación, durante sus últimos años, de un aparato semi-corporativo donde los partidos políticos (actores principales del pacto de Punto Fijo) fueron perdiendo poder frente a otros poderes y grupos sociales.
} 
Con la elección de los primeros alcaldes, en 1989, se lleva a cabo una pequeña revolución de la relación entre lo local y lo central. Una de las iniciativas pioneras, el presupuesto participativo de Caroní, es la experiencia más concreta de un proyecto local de construcción de un "gobierno de participación popular" (Harnecker 1994: 34) basada en la crítica a las élites y en la recuperación democrática. En este mismo periodo de transición nacen otros espacios de participación y de exploración de nuevas formas de esquivar la inercia política de esos años.

En Caracas, la experiencia de la Asamblea de Barrios, que comienza en el transcurso de 1991, es interesante en varios planos ${ }^{7}$. Primero, aunque de existencia fugaz, ya que se disuelve en 1993, siempre está presente en los relatos de los militantes de izquierda. Así lo afirma Miguel Barreto, militante de una organización popular de la parroquia La Vega, cuando comenta que:

"el trabajo que se hace aquí en Las Casitas tiene antecedentes. Esto no depende ni se activa con el proceso revolucionario. Nosotros podemos decir con mucha humildad que esto ha dado aportes al proceso, porque muchas cosas que tú ves ahí [en las políticas de gobierno] vienen de discusiones que datan de hace mucho tiempo, incluso plasmadas en las Asambleas de Barrio" (Entrevista realizada el 04/08/07).

Además, porta el germen de un cierto número de proyectos y de estructuras muy parecidas a los marcos actuales de la política - oficial y militante ${ }^{8}$ - chavista. Por último, nos puede decir mucho sobre las

${ }^{7}$ Las Asambleas de Barrios de Caracas nacen como iniciativa de la mesa de pobladores reunida en el Encuentro por la Rehabilitación de los Barrios del Tercer Mundo realizado en la ciudad de Caracas entre el 24 y el 29 de noviembre de 1991. Como espacio de articulación popular, las Asambleas de Barrios de Caracas (ABC) estaban lejos de toda concepción relacionada a las organizaciones no gubernamentales (ONG). Según uno de sus militantes: "las ABC, a diferencia de las Asociaciones de Vecinos, se visualizaban como un movimiento de participación popular. Como un proceso de auto-organización cuya raíz histórica son los grupos de izquierda. Mientras la perspectiva de las asociaciones de vecinos y ong's era reformista, las ABC apuntaban a ser revolucionarias. La diferencia de clase está que mientras unas [las $\mathrm{ABC}]$ se organizaban en barrios las otras se organizaban en urbanizaciones" (Caracas, 12/02/2010) (Ver. Denis, 2001 ; Rey Torres, 2011).

${ }^{8}$ De las discusiones, debates y reflexiones que se dieron en el contexto de las ABC, las políticas relacionadas a, por un lado, la gestión de servicios públicos como el agua, el transporte, la electricidad, y por el otro, a las políticas de reconocimiento y rehabilitación integral de Barrios y la regularización de la tenencia de la tierra urbana, se desprendieron programas que -históricamentedurante la década de los noventas y sobre todo a partir del 2002 (con el decreto 1666 de regularización de la tenencia de la tierra urbana) formaron parte de las agendas y programas, en primer lugar, de gobernaciones y alcaldías con representantes de "izquierda" (la Causa R principalmente), y en segundo lugar, parte de las políticas públicas dirigidas a las organizaciones de base que luego se 
formas de elaboración de los nuevos repertorios de acción colectiva que se encuentran enraizados en la participación democrática de los barrios autoconstruidos, que representan más de la mitad del hábitat urbano.

La Asamblea de Barrios, en aquel contexto, representa el primer banco de pruebas de un movimiento social urbano popular y de la reaparición de las luchas urbanas con vocación hegemónica (Denis, 2001). Desde el punto de vista del paradigma colectivo de las reivindicaciones, se le da importancia al acceso a la propiedad de los terrenos, a la rehabilitación física de los barrios, a la cogestión de los servicios públicos y al reconocimiento de los “autogobiernos locales" (Antillano 2005). Durante todo este periodo, las consignas son: "no queremos ser gobernados, queremos gobernar" o "democracia de la calle" (Denis 2001).

Varios son los factores que intervienen en la explicación de la transformación del movimiento social urbano; de estos podemos subrayar su naturaleza: en primer lugar, la inserción de las reivindicaciones propiamente urbanas en la agenda del militantismo de izquierda, que hasta ese momento las consideraba reformistas. Luego, el descrédito de los dos órganos de representación más importantes de la época, que funcionaban como medio de captación y de redistribución de renta en ciertos casos: el partido político y la Asociación de Vecinos. Por último, y tal vez lo más significativo en nuestra opinión, la explosión social del "Caracazo" (1989), que surge en la confluencia de todos los episodios conflictivos y transgresores del orden público desde 1958, y que deja su huella en la historia venezolana reciente. De hecho, todos los actores del movimiento progresista van a encontrar en él un espacio común de resonancia.

En medio de este periodo de una gran riqueza en cuanto a innovaciones y creatividad política, aparecerá otro tipo de organización bajo la influencia del Estado: las Asociaciones de Vecinos. Estas se insertan en un proceso de descentralización iniciado en los años 80 , y van a jugar un papel central en la redefinición de las reglas del juego político, tanto en el rechazo hacia el modelo democrático propuesto como en las renovadas formas de acceso a los recursos políticos y a los bienes materiales. Su nacimiento se remonta a los años 30, pero 
realmente se institucionalizan en el transcurso del primer gobierno electo, en 1958. Asistimos, por decirlo de alguna forma, a una "invasión" de los barrios marginales y periféricos por parte del Estado y los partidos políticos. Es así que se observa un fenómeno de sumisión de las organizaciones populares urbanas al Estado en una relación de "articulación dominada" (Hurtado 1991), que resulta en la profesionalización de los líderes locales. Lo que sigue muestra la sucesión de luchas de influencia de los partidos políticos para ganarse los favores de las organizaciones barriales. En 1971, ven la luz las “asociaciones de propietarios y residentes" y se convertirán en una especie de "vanguardia administrativa de los habitantes" (Salamanca 1987). Estas responden a objetivos precisos de lucha contra el deterioro del medio urbano, enfrentan el crecimiento inmobiliario descontrolado posterior a los años de despegue económico, y circunscriben su acción a una forma de lobbying dirigido hacia las instituciones y las empresas privadas.

Por último, las Asociaciones de Vecinos se definen abiertamente como apolíticas. Para reforzar su área de acción, como también su influencia en tanto que movimiento social (incluso si rechazan esta denominación), se van a reagrupar en una federación (FACUR) y ganarán su prestigio atacando la estructura política y administrativa de la municipalidad y logrando la obtención de un estatus de "representante de la sociedad civil". Sin embargo, estas organizaciones van a fragmentarse progresivamente en el transcurso de los años 90, debido tanto a la acción externa de los partidos que buscan asentar su influencia en ellas, como al hecho de la dispersión interna de sus acciones, demasiado centradas en los aspectos locales. Hoy en día, su capacidad de acción ha declinado considerablemente (inclusive si han podido reconstruirse en torno a las estructuras provistas por el gobierno bolivariano), sin desconocer que en ciertos casos sigue siendo bastante importante. Para algunos, "la democracia participativa comenzó a funcionar a través de la acción de los habitantes" (Salamanca 1987). En consecuencia, incluso si las fronteras de la relación clase-dominación son tan claras como en las

\footnotetext{
${ }^{9}$ Ese estatus, en tanto modo de operación de efectividad simbólica, permitió que las Asociaciones de Vecinos se constituyeran como un elemento creador de sentido, en el proceso constitutivo de la Comisión Presidencial para la Reforma del Estado (COPRE) en diciembre de 1984 (Lander, 2006).
} 
otras formas de movilización, las Asociaciones de Vecinos no dejan de constituir un amplio movimiento que atrajo a múltiples sectores de la sociedad venezolana ${ }^{10}$. Sin embargo, los actores chavistas de la participación siempre hacen énfasis en las diferencias irreconciliables entre la participación durante la "cuarta" y la "quinta"" República, como lo destaca una entrevistada, habitante de un barrio de Caracas, y militante del Frente Francisco Miranda:

"Porque con las AV había muy poca participación, la gente participaba, pero de una manera apática o para recibir las constancias de residencia que atestaba el sello de la asociación que yo vivía en ese sector. La gente no participaban por el bienestar colectivo, nunca se preocupaban de la iluminación del sector o de la recolección de basura"

Francia, Barrio Obrero, Manicomio, Caracas, 16/01/2007

\section{LA PRÁCTICA POLÍTICA DE LA PARTICIPACIÓN: LOS CONSEJOS COMUNALES}

Cuando en 1998 Hugo Chávez menciona las políticas a emprender en materia de participación, subraya la naturaleza descentralizadora del proyecto bolivariano, que no puede llevarse a cabo sin una "desconcentración” de los poderes públicos. Antes de 2006, los Consejos Locales de Planificación Pública (CLPP) eran reconocidos como agentes de referencia de la organización y participación a escala municipal, parroquial y sectorial. El 7 de abril de 2006, la Asamblea Nacional de Venezuela, adopta la ley de los Consejos Comunales. Esta se inscribe en el marco general del lanzamiento, que

\footnotetext{
${ }^{10}$ Así lo afirma Edgardo Lander (2006) en su texto Acción Social, efectividad simbólica y nuevos ámbitos de lo político: "Es a partir de estas últimas organizaciones, inicialmente surgidas en sectores de la clase media alta, que en los años setenta se comienza a desarrollar un movimiento ciudadano con características distintivas, que se va extendiendo a urbanizaciones de sectores menos pudientes y también a algunos barrios de menores ingresos, no sólo en Caracas, sino así mismo en otras ciudades del interior del país. [...E]n esta extensión y perfilamiento de los rasgos propios de este movimiento vecinal juega un papel decisivo la acción coherente y bien organizada y pertinaz de una pequeña minoría. Es ella la que opera como conformador de sentido, imponiendo incluso su propio lenguaje, símbolos y estilo; ello no ocurre de una manera casual, sino como estrategia explícita del propio movimiento" (Lander, 2006: 45-46).

${ }^{11}$ Para Hugo Chávez, el chavismo se desplegaba como un movimiento histórico que refundaría las bases del estatuto republicano venezolano. En ese sentido, la quinta república fue el enunciado que operó como mecanismo de distinción entre el período de la democracia puntofijista o cuarta república (1958-1998) y la quinta república que se inauguraba con la nueva Constitución.
} 
tiene lugar durante el discurso de investidura del segundo mandato de Hugo Chávez, el 8 de diciembre de 2007, de un sexto poder, componente de la "nueva geometría del poder": el poder popular ${ }^{12}$.

Respecto a la ley, los Consejos Comunales son concebidos como unidades territoriales de base, áreas comunales que abarcan de 200 a 400 familias en las zonas urbanas, entre 30 y 40 en las zonas rurales y 10 a 20 en los territorios indígenas. Deben formarse a partir del tejido organizacional preexistente; incluyendo, organizando y articulando a los diferentes comités, las cooperativas y las asociaciones socioculturales ${ }^{13}$. Estas unidades de organización popular son concebidas con la finalidad de resolver los problemas de base de la comunidad (salud, acceso al agua potable, propiedad de la tierra, gas y electricidad, infraestructura, etc.) y abrir espacios de práctica política.

Podemos definir dos formas de legitimación de los marcos estructurales de los Consejos. Son constituidos por los voceros, elegidos por los electores del sector mayores de 15 años, y avalados por la Asamblea de Ciudadanos y Ciudadanas. Los primeros forman el organismo ejecutivo encargado de organizar el "ciclo comunal" a través de cada uno de los comités de trabajo ${ }^{14}$, los miembros de los órganos financieros y de contraloría social. La segunda representa la instancia y la autoridad de referencia, a través de la cual se toman las decisiones y se establece el "poder popular". Una vez que el consejo comunal se haya formado, sus miembros deben presentar un proyecto socio-productivo, de renovación, de infraestructura, etc., que será aprobado en asamblea.

Una de las grandes temáticas que acompaña el discurso gubernamental de la participación, y a fortiori sobre los Consejos Comunales, se funda en la "corresponsabilidad" ciudadana. Este no es un término nuevo en el lenguaje institucional venezolano, puesto que ya aparece en el programa de descentralización de fines de los años 70 y toma un

\footnotetext{
${ }^{12}$ Esta compleja aplicación del proyecto global contenido en la fallida reforma constitucional de 2007 establece un redimensionamiento de las relaciones político-territoriales y del espacio socio-administrativo. Otorga un lugar preponderante a las "comunidades organizadas" en la búsqueda de soluciones a los problemas frente a los cuales deben responder las instituciones locales. A partir de la puesta en marcha de los "cinco motores de la revolución", el poder comunal es integrado en el panteón de los poderes públicos. Los Consejos Comunales están en el centro de este proyecto.

${ }^{13}$ Esto en principio. Los Consejos comunales se convirtieron más bien en espacios de representación de diferentes áreas sociales y necesidades de la comunidad sin ser el gran modelo de unión que se preveía. La Comuna tendría como lo veremos más adelante ese papel.

${ }^{14}$ De manera exhaustiva, la ley preconiza una decena de áreas temáticas como: la salud, la protección social, la infraestructura, la energía, la cultura, la alimentación, etc.
} 
carácter definitivo en la Constitución de 1999. Concretamente, se presenta como la respuesta a la pregunta: ¿cómo la comunidad y el Estado pueden trabajar en común para encontrar soluciones a los problemas locales? En resumen, se trata de implementar estructuras perennes de comunicación con las instituciones con la finalidad de provocar una captación, una atracción que se ejerce de arriba hacia abajo.

Esta idea se difundió de manera tan amplia en el orden jurídico y político que los Consejos Comunales han debido asumir las características de una institución, con el fin de instaurar esta trama comunicacional entre las comunidades y el Gobierno. En este sentido, son aparatos de comunicación política, y no instituciones completamente estabilizadas. Ahora bien, la corresponsabilidad adquiere una función de doble contenido: el derecho a la toma de decisiones en temas locales en contrapartida de lo cual las comunidades tienen un deber de participación y de acción para el bien público. Es también lo que subyace en la reforma que da origen a la Ley Orgánica de los Consejos Comunales que entra en vigor el 29 de diciembre de 2009.

Un elemento importante en el esbozo de la nueva semántica política que se expresa en esta reforma, es la emergencia del carácter ideológico de los consejos como motores de construcción del socialismo, de la misma manera que procede a la introducción del concepto de "gobierno comunitario", cuando la versión anterior sólo hacía mención del "poder popular". Es obvio que, la tímida política de los inicios del primer Gobierno de Chávez en materia de políticas participativas se modificó ampliamente al entrar en contacto con la muy dinámica realidad de la participación popular. Este "giro" participativo que se inicia en 2004 volvió a impulsar toda una gama de proyectos en los cuales los consejos comunales representan la piedra angular. Abre el camino a una consolidación de los esfuerzos participativos a una escala política e institucional. 


\section{HACIA LA CONSTRUCCIÓN DE LAS COMUNAS Y EL ACERCAMIENTO ENTRE LA TEORÍA RADICAL DE LA DEMOCRACIA Y LA REALIDAD SOCIAL}

La figura de la Comuna aparece en la reforma de la ley de los Consejos Comunales junto a la de las "ciudades socialistas", y busca llegar al ideal marxista de la "forma política finalmente encontrada" (Marx 2008, p.58). El Presidente en varias oportunidades desde el 2006 hace público su plan para la amplificación territorial y decisional de los dispositivos de participación: "Los Consejos Comunales no pueden quedarse en la superficie ni en el simple hecho de hacer un proyecto, presentarlo y que nos den un recurso: ese no es el objetivo fundamental, necesitamos que se vayan estructurando en una verdadera célula-motor en cada comunidad"15, y "Yo pienso en una suerte de federación de los consejos comunales"16.

Si las instituciones son la cara visible de la participación y del proyecto global que lo respaldan como vía de elaboración de nuevas relaciones políticas, y más allá de poder, la dimensión territorial no debe perderse de vista. Es un elemento que se hace presente en el conjunto de los discursos y de las prácticas de los actores, particularmente desde la idea de la "reterritorialización". Esta expresión es común en el discurso político de las izquierdas latinoamericanas que se orientan hacia la ruptura de los esquemas impuestos por la historia colonial y la dominación socio-económica. En Venezuela, este término ha sido sustituido por el concepto, heredado de la geografía radical, de "nueva geometría del poder". En esta redefinición de las relaciones de significados y prácticas, lo que prevalece en definitiva es la capacidad de los individuos y grupos de asumir varios niveles de representación (el barrio, la parroquia, el municipio, la nación) en una visión posneoliberal y decolonial.

No obstante surge un problema central en la direccionalidad política que se da al impulso de las Comunas, en tanto extensión del proyecto participativo de autogobierno: mientras más extendido sea el territorio, mayores son los intereses en juego. Entonces, ¿bajo

${ }^{15}$ III Gabinete Móvil de Consejos Comunales. Tucupita, estado Delta Amacuro, 20 de mayo de 2006. ${ }^{16}$ Aló Presidente, 31 de octubre del 2010. 
qué aspectos podemos entender la tensión entre la territorialidad y el proceso de organización? ¿Cuál es la relación entre el territorio y el espacio político? Y subsecuentemente, ¿entre las desigualdades sociales y la participación territorial?

Si observamos las características de la democracia participativa en otros contextos, surge la interrogante de ¿cómo evitar que una "democracia de proximidad" fundada en la pertenencia a un lugar y en los saberes que derivan, se convierta en una "democracia de enclaves", y que, debido a esto, los ciudadanos se conviertan solo en habitantes o, más aún, en "habitantes profesionales" (Blondiaux, 2008)? De ahí, ¿cómo la ampliación territorial de la participación puede ser percibida como la emanación de una "democracia radical" (Laclau y Mouffe, 2009)?

El proyecto de "democracia participativa y protagónica" busca incidir en la dispersión de los centros de poder y en el redimensionamiento de las decisiones a escala local. Para esto, el consejo comunal aparece como un instrumento de primera línea si va acompañado de una extensión territorial. La Comuna, trazada bajo el modelo ideal de la Comuna de París, busca conferir más prerrogativas a las comunidades ofreciéndoles la posibilidad de asociarse con el fin de dotarse de poderes más importantes (ampliación de los proyectos, nacionalización y homogeneización de los territorios, capacidad productiva).

Antes de que la Ley de Comuna fuese adoptada a fines de 2010, comienzan a crearse a través de todo el país las "comunas en construcción". Nadie logra entender bien el alcance del proyecto, sin embargo Chávez en persona se desplaza para oficializar sus creaciones. Esto provoca algo de confusión y reinstala la idea de un para-institucionalismo que refuerza la capacidad organizativa de las comunidades. Es así que la Comuna se convierte en la forma predominante de ascensión de la participación.

Los efectos del hiperlocalismo provocados por el espacio de acción de los Consejos Comunales condujeron a prever la ampliación de las prerrogativas colectivas, primero, en términos de territorio y, a continuación, de funciones. Desgraciadamente esta operación de maximización de la participación no corrió la misma suerte en todas las experiencias. En efecto, algunas Comunas parecen gozar de 
mucho apoyo por parte del Estado, mientras que otras deben bregar en medio de la burocracia, del papeleo y del laberinto administrativo que dispuso el Estado en vista de "legitimar" o legalizar esas organizaciones. En otros términos proporcionarles un barniz institucional, lejos de la idea de movimiento social o, incluso, de poder popular. Igualmente, esta se convirtió en el sello político de la participación, ya que a través de ella los consejos comunales se transforman en actores políticos de pleno derecho, como lo afirmaba Chávez en una alocución en febrero de 2009.

Los actores locales de esa transformación política tienen un sentimiento equivalente:

"Yo pienso que vamos a tener una personalidad jurídica mejor definida [vamos a poder] formar gobiernos parroquiales en nuestras comunidades, para no depender tanto de las instituciones, tener un presupuesto propio, una forma propia de administrar los recursos en beneficio de la comunidad: pienso [que la reforma de la ley va] a ayudar a consolidar este trabajo comunitario, no sólo al interior en las comunidades sino también a través de las comunas. Por fin tendremos el poder de controlar nuestro desarrollo" Jimmy, miembro del CC San Antonio, Candelaria, Caracas, 11/11/2009

Entonces, la democracia participativa se presenta como un instrumento de modernización, para rebasar el carácter tradicional y patrimonial de las prácticas políticas, consideradas atrasadas en comparación con el sistema "occidental". Pero la construcción de las Comunas revela también las tensiones al interior de la sociedad organizada, que percibe su distancia con respecto a los que no participan. De esta forma, se observa el desarrollo de disparidades en los territorios que se supone deben encarnar una cierta homogeneidad, en particular en los barrios. Como lo evoca un habitante no-politizado del 23 de Enero, barrio de gran renombre en las luchas sociales de la capital, y feudo del "chavismo radical": 
"El tema de las comunas y de los consejos comunales es percibido más bien en un marco político que social, ya que en su gran mayoría se trata de pequeños grupos que no llegan al conjunto de la sociedad". Wilfredo, CC Bloque 17, 23 de Enero, Caracas, 18/07/2011

\section{CULTURA POLÍTICA PARTICIPATIVA}

Antes de entrar en el corazón de nuestra contribución empírica, queríamos establecer el marco normativo de nuestro trabajo. A la lectura de nuestra experiencia en sectores populares de Caracas, encarnando el giro participativo que sustenta la Revolución Bolivariana, consideramos que existe una estabilización relativa del movimiento social chavista. Este se define en la escritura diacrónica de una "cultura política participativa" y la conformación progresiva de una sociedad movilizada. Explicaremos a continuación por qué.

Nos parece característico del periodo 1999-2014 el surgimiento de la participación como una materia maleable, inestable y transmitida por los actores en interacción que van, entonces, a adaptar su definición del bien común y del bien público, por un lado, y a reformular experiencias heterogéneas o conflictivas, por el otro. En muchos casos, estas interacciones y reformulaciones han sido producto, tal y como lo ha demostrado Merklen (2005), para la experiencia Argentina, de procesos de mutación y reconfiguración del mundo del trabajo en el contexto neoliberal. Así, las redes sociales, políticas y económicas que van surgiendo en el proceso de participación, tienden a anclarse territorialmente generando disputas, negociaciones y/o mediaciones alrededor de, por ejemplo, las dimensiones y modalidades de las estructuras de participación, los procesos de transferencia de recursos por parte del Estado, políticas de asistencialismo, entre otros. Si bien las transformaciones de la cultura política, como lo han demostrado algunas investigaciones sobre las dinámicas de participación en latinoamérica (Ver. Álvarez et al., 1998; Mato, 2004; Lander, 2006, entre otros), tienden a reconfigurar las narrativas alrededor de la democracia, las dinámicas de participación, ciudadanía, etc., también es cierto, que genera recon- 
figuraciones en las dinámicas materiales de las condiciones de vida, y a su vez que generan nuevos sistemas de relación, interacción y construcción de vínculos sociales. De manera que si el uso de la noción de cultura política parece problemático, no por ello deja de ser una herramienta -sin duda versátil y proteiforme, que amenaza con volverse contra el observador- que puede permitirnos abordar cuidadosamente los "sistemas de significación" - como los resaltados por Clifford Geertz (1992) - puestos en práctica en todo fenómeno cultural, así como también puede permitirnos descubrir el sistema simbólico subyacente en las acciones de los individuos en una situación o grupo determinado.

En numerosas experiencias estudiadas durantes los últimos diez años del proceso político venezolano, nos ha aparecido importante la influencia, interpenetración o hasta imposición de las directrices del partido chavista, el PSUV, con las organizaciones no directamente políticas (Consejos Comunales). Sin generalizar el alcance de dicha relación evidenciamos cierta afinidad electiva de actores pertenecientes al mismo "espectro político", esto es el chavismo, que se expresa en el rechazo de cualquier imposición, o la negociación y colaboración entre grupos, y en última instancia, la indiferencia. Un observador del sistema de organización de los barrios hablaba en los años noventa, es decir antes del advenimiento del chavismo, de una "doble identidad" de habitante y militante. Ésta se cristalizó, posteriormente, en favor de la segunda dado el movimiento de captación clásico del partido, el cual no podía “aceptar que prevaleciera su condición de vecinos, así como los intereses vecinales sobre las líneas y prioridades de la organización, más aún si estaba en el gobierno » (Trigo 1995: 98). Ello representa una premisa importante para apuntalar las características de una desconfianza recíproca que aún perduran pese a un sistema de oportunidad política que intenta aglutinar todas las iniciativas bajo el lema de la unión política y del cambio social. Sin embargo, a pesar de esta desconfianza recíproca, los procesos de interlocución e interpelación, entre el Estado y las estructuras de participación, parecieran no encajar en los análisis prescriptivos y normativos que tienden a caracterizar, dicha relación, a partir de, por ejemplo, las nociones de cooptación y clientelismo (García-Guadi- 
lla, 2008; Vargas, 2010). Por el contrario, las modalidades de la relación entre comunidad/Estado y comunidad/partidos políticos, ponen en tensión dichas nociones y generan la necesidad de establecer miradas que busquen aproximarse al análisis de las relaciones de poder, colocando en tensión tanto el nivel estructural como el agenciamiento de los actores, y permitiendo así revelar las diversas modalidades existentes en el campo de interlocución estratégico entre el Estado y las estructuras de participación. En el terreno, estas fricciones se traducen en reiteradas oportunidades por la aparición de descalificaciones por "disidencia" anti-revolucionaria o a su vez por el desmoronamiento, la "desintegración" de la dinámica comunitaria.

Sin embargo, la cultura política participativa -anclada en la historia reciente de las movilizaciones populares en Venezuela- manifiesta un proceso de politización sui generis que no se define por la sola pertenencia a un partido político, sino por una socialización participativa contenida en las leyes, el territorio y el trabajo social ceñido por liderazgos locales. Lo que llamamos la socialización participativa refiere a las actividades que se elaboran en diferentes escalas de la acción colectiva local: papelógrafo, perifoneo, elaboración de proyectos, organización de asambleas, de mercados populares, etc. Es, por decirlo así, una transposición indirecta de lo político en el espacio social-comunitario.

Desde ese punto de vista, es pertinente recalcar la reivindicación emanada de la sociedad participante hacia el resto de la comunidad al hecho de que no existen fronteras entre las necesidades básicas (alimentación, vivienda, salud, infraestructura, etc.) y la atribución de un derecho a participar y actuar en el terreno político.

La "democracia participativa y protagónica" resalta el peso de la política sobre la disposición general de los actores en la participación. A su vez, la participación refuerza el alcance político de las discusiones y prácticas que se vienen desarrollando en su seno. La cultura política tiende a sedimentar los aprendizajes productos del arraigo e institucionalización de la participación. Anuncia un nuevo repertorio de aparición democrática que se desliza en los pliegos de una sociedad movilizada, proclive a un cuestionamiento profundo 
y continuo de las relaciones sociales y políticas. Estos dos procesos son, según nuestro entendimiento, característicos de una subjetivación política propia a la historia política de la participación en Venezuela, en un marco extendido y no solamente individual o militante.

La lucha política de los años 70 a 90 - sea en el sindicalismo, o sea en los partidos de izquierda o grupos semi-clandestinos - en la periferia urbana autoproducida ("barrios"), el tránsito de una vida hogareña a una vida pública local, o la del ex-delincuente convertido en líder local ${ }^{17}$, la cuestión del trabajo y el compromiso social son las tantas apariciones, détournements e interrupciones que establecen al sujeto político, históricamente marginalizado, invisibilizado y confinado a la esfera productiva y de la sobrevivencia. A su vez, la participación es también el marco de absorción de las dificultades y aporías de la democracia directa: hay sujetos políticos porque todos no pueden serlos. Por ello, la democracia participativa no es completamente un "movimiento social", por demás extendido y heterónomo en sus relaciones al Estado. Correspondería más bien a la puesta en práctica del "derecho a tener derechos" enfatizado por la tradición arendtiana (Alvarez et al., 1998) y reactualizada en el "giro pragmático" (Pogrebinschi, 2013) de la política latinoamericana de los últimos años.

\section{UNA REALIDAD EN TRANSFORMACIÓN: NUEVOS AC- TORES DE LA PARTICIPACIÓN}

En la actualidad, las dinámicas de la participación popular en los consejos comunales y las comunas, vienen transitando un proceso de transformación asociado a la emergencia de nuevos actores dentro de los espacios participativos. En este sentido, las trayectorias que durante los primeros años del proceso de cambio social propuesto por el chavismo, se encontraban asociadas a historias de militancia y activismo (partidos o grupúsculos de izquierda, asociaciones

\footnotetext{
${ }^{17}$ Sobre este punto que desarrollaremos a continuación, procura interrogarse sobre los procesos de "ciudadanización" y zonas grises de la participación popular puesto en énfasis en los trabajos sobre la Argentina de los años 80 a 2000. Estos recalcan las peculiaridades de la economía de la subsistencia en contextos de crisis económicas, y las fluctuaciones de los "poderes territoriales" (Auyero, 2007; Ossona, 2014).
} 
de vecinos) previas a 1998 o forjadas al calor de la participación en estructuras a partir del 2003 como los Comités de Tierras Urbanas, de salud y Mesas Técnicas de Agua, comienzan a confluir con actores políticos emergentes que otrora no participaban y/o eran excluidos de las instancias de participación. Esta aserción se fundamenta en una investigación en curso desarrollado por nuestro equipo, quien trabaja simultáneamente en diferentes sectores de la capital, y en territorios diversamente organizados. Lo que surge de ello, es la aparición de figuras nuevas en el marco de la política popular encarnada por el nacimiento y fortalecimiento de organizaciones sociales tales como los Consejos Comunales y las Comunas.

En este sentido, de la misma manera que existen disparidades territoriales, también surgen disparidades en el seno de las mismas comunidades. Sin abordar los clivajes políticos (entre chavismo y oposición, principalmente) que pueden permear y entorpecer los procesos de organización orientados hacia la obtención de resultados materiales concretos para el bien común local, aparece una línea de separación entre grupos formados en base a una aceptación política (militantes del partido del gobierno, luchadores sociales de vieja data, funcionarios públicos) y nuevas caras de una política en permanente formación.

Figuras marcadas por el surgimiento de actores jóvenes y masculinos, entre 20 y 30 años, sin trayectoria política, socialmente desafiliados y cuyas trayectorias se encuentran relacionadas, o bien al trabajo informal y precarizado, o a su participación en actividades ilícitas. Por un lado, los llamados "colectivos" que tienden a ser grupos con características para-policiales, pero apoyados por entidades gubernamentales (Hanson, 2014). Por el otro, los sindicatos de construcción, que suelen ser uno de los mayores empleadores de la mano de obra masculina y joven de los barrios populares. Si bien estos dos tipos de organización no son nuevos - aunque en el caso de los "colectivos", han tendido a multiplicarse en los últimos dos años - recae sobre ellos la característica común de tener mala fama. Sin embargo, a la luz de nuestras observaciones, aparecen como actores de primer plano y en vía de acercamiento a la modalidad organizacional encarnada por las Comunas. 
Recientemente los "colectivos", en tanto modalidades de organización, han venido atravesando un proceso de reconfiguración respecto a sus propiedades constitutivas. Desde el calco de las viejas Unidades Tácticas de Combate (UTC), pasando por su representación como "vanguardias" políticas de un determinado ámbito geográfico originada en las luchas sociales de los noventa, los colectivos - en la actualidad - actúan en un campo que se moviliza entre el despliegue de prácticas para-policiales (que mantienen a raya a la delincuencia y el narcotráfico, por ejemplo) y la apuesta por la construcción orgánica dentro del ámbito comunitario.

Este proceso de reconfiguración, con la emergencia de nuevos actores políticos dentro de las comunidades, genera disputas y disparidades -como se dijo anteriormente - que atraviesan transversalmente los marcos materiales (disputas respecto a los canales de distribución de la renta petrolera) y simbólicos (reconocimiento, crédito moral) de las luchas por el bien común. En un sector donde se desarrolla nuestra investigación se ha observado este proceso de reconfiguración. Representándose a sí mismos como un movimiento relacionado a la promoción cultural y deportiva, el colectivo ha desplegado una diversidad de prácticas que, desde su perspectiva, se traducen en esfuerzos por la construcción del socialismo y la mejora del ámbito comunitario. Apuestan a la pacificación de los territorios, y al "desplace"18. En otro contexto, dispositivos de seguridad y vigilancia (guardias, rondas, circuitos cerrados, entre otros) en conjunción con modalidades de regulación social y de la "convivencia" (horarios para las fiestas, volumen de la música, desplazamiento después de determinadas horas en el territorio en el que se inscriben) han generado formas de administración que expresan, por ejemplo, mecanismos para-estatales de administración de la justicia.

Aunque los principios universales de las organizaciones que hacen vida en la comunidad (construcción del socialismo, mejora del hábitat comunal, entre otras) permanecen intactos en tanto significantes, las

\footnotetext{
${ }^{18}$ En un contexto exponencialmente marcado por fronteras simbólicas y culturales asociadas a prácticas violentas, el tránsito de una frontera hacia otra con relativa ausencia de riesgo, es comúnmente denominado como "desplace". En este sentido, la posibilidad del "desplace" se encuentra asociada, entre otras cosas, al volumen de capital social (violento o no) de quien pasa de una frontera a otra, y por ende a una cierta forma de acumulación proporcional de capital territorial.
} 
modalidades de organización de las prácticas políticas en la cotidianidad divergen no sólo en términos de tácticas y estrategias, sino que también despliegan una diversidad de posicionamientos que problematizan, por ejemplo, la efectividad material y simbólica de la práctica política de militantes que provienen de experiencias previas y la gestión de los recursos provenientes de las instituciones del Estado, a través de las distintas figuras de financiamiento para proyectos locales.

Así lo expresa un integrante del Movimiento por la Paz y la Vida $^{19}$ de una de las comunidades estudiadas, cuando afirma que:

« tú sabes que [los consejos comunales] no hacen nada, son unas ratas, se quedan con todo, con los reales, y no hacen nada... Por ejemplo Fulana, qué hace? Nada. Tiene un poco de pintura en la casa y no la usa. Nosotros en cambio conseguimos la pintura por otro lado, con el gobierno, y pintamos las escaleras. Dígame Mengano, que tiene toda la vida ahí y no hace nada. Hace como un año le dieron los papeles para solicitar una silla de rueda a un señor, y no ha hecho nada. Nosotros en pocos meses se las conseguimos, y de maldad y todo se la vamos a entregar al señor, para que vean que nosotros sí hacemos, si la movemos. [...]Todo se va a voltear, ya no es lo mismo. Aquí todo cambió. Nosotros somos los que estamos con el gobierno. ¿No te das cuenta? Ahora aquí mandamos nosotros" (Conversación informal, 20/02/2014).

Estas disputas y disparidades que en apariencia parecieran poner de manifiesto distinciones asociadas a trayectorias históricas dentro de la comunidad y brechas generacionales, expresan desplazamientos transversales entre diversas formas de organizar la práctica política y las formas en cómo estas traducen los principios universales de la lucha. ¿Qué se gestiona, quién y cómo lo gestiona y cuál es la temporalidad de la gestión? ¿Cuáles son los logros obtenidos, qué beneficios han aportado para el mejoramiento de la comunidad? son algunas de las interrogantes que caracterizan estas disputas y disparidades, no sólo en ${ }^{19}$ Organizaciones sociales promovidas « desde arriba » por el Ministerio del Poder Popular para el Interior y Justicia. El objetivo de estas organizaciones era el de promover una cultura de paz, a través de -principalmente- la construcción de espacios de paz y convivencia (canchas). El Movimiento que estudiamos se autodenominaba "colectivo". 
términos de capital simbólico (quién obtuvo o generó tal o cual proceso, y es reconocido por ello), sino que también expresan desplazamientos verticales con agentes e instituciones del Estado (a través de qué institución o qué contacto) que dan cuenta de una variedad de volúmenes de capital social que ponen en juego, tensionan y potencian las principales demandas y reivindicaciones de la comunidad.

En este sentido, los colectivos tienden a incorporarse dentro del campo político y del ejercicio "institucionalizado" del poder dentro de la comunidad: los Consejos Comunales. Bien sea a través de alianzas y articulaciones, a través de la postulación de sus miembros a las distintas vocerías, o a través de la constitución del Comité Promotor del Consejo Comunal, los colectivos generan campos de interlocución e interpelación en los que tanto las formas propias de organización de sus prácticas (el ejercicio potencial de la violencia) y los marcos estables de la participación dentro de los consejos comunales (reuniones, asambleas y elecciones), dialogan y se disputan formas de otorgarle sentido a las dinámicas cotidianas que se despliegan en, por ejemplo, la planificación y la ejecución de proyectos.

Algo similar sucede con la práctica desplegada con los sindicatos de la construcción en relación con los Consejos Comunales y los procesos de construcción de la Comuna. Con modalidades de ingreso al campo político y del ejercicio institucionalizado del poder dentro de la comunidad, los sindicatos de la construcción tienden a relacionarse con los Consejos Comunales y las Comunas a través de, por un lado, los espacios legitimados de reunión, y por el otro, a través de demandas relacionadas a, por ejemplo, ingreso de personal y mejoras salariales, en una obra que para ese momento se esté desarrollando en el territorio comunal. Esta situación ha sido observada en dos de los sectores que hacen parte de nuestra investigación en curso.

Símbolo de una entrada nueva al campo político-comunitario, la Unión Bolivariana de Trabajadores (UBT)-El Equipo, asiste a reuniones de la Comuna X en Antímano, asume una postura pacífica en la obtención de puestos de trabajo que dista del uso usual de la coacción violenta y rasgos delictivos que muchos de estos grupos adoptan. Usan un discurso politizado del "poder popular", aún siendo compuestos 
por jóvenes anteriormente relegados, y no inscritos en el tradicional campo laboral (sea porque trabajaban en el sector informal, o eran previamente delincuentes). La socialización de ellos pasa por el trabajo y la forma organizada encarnado por el gremio obrerista de la construcción, muy distintos de otras áreas obreristas. Sin tener un anclaje territorial tan localizado como los Consejos Comunales o la Comuna - por su naturaleza su influencia tiende a (y debe) desplegarse en toda la ciudad - reivindican el bienestar de la comunidades (y de la clase obrera) como eje orientador de sus acciones.

Respecto a la segunda modalidad de ingreso y diálogo señalada, se pudo observar que las disputas relacionadas a la construcción de las obras (ingreso de personal y mejoras salariales) se encontraban mediadas por otras modalidades de organización existentes en la comunidad. En este caso particular, en la parroquia Santa Rosalía, las disputas se generaron a partir de las demandas de una banda de delincuentes que hace vida en el sector. Lejos de ser percibido como un espacio de articulación de los intereses de clase frente a la explotación patronal, el sindicato -en este caso- parecía operar como una extensión de las dimensiones territoriales y relacionales de estos actores caracterizadas por la extracción de renta por medios ilícitos. En este sentido, se produce nuevamente un desplazamiento transversal que tensiona y confronta dos grupos de agentes existentes en la comunidad. Disputas que exigían un manejo efectivo de los recursos financieros relacionados a la ejecución de proyectos $\mathrm{y}$ donde se demandaban una cantidad específica de puestos de trabajo, cantidades específicas de remuneración y bonos adicionales una vez finalizada la obra. Disputas que por demás, se encuentran mediadas -nuevamente- por la emergencia de actores disruptivos (que tienden al despliegue de prácticas violentas) que tensionan las modalidades de interlocución y participación dentro de la comunidad.

Finalmente, para ambos casos, una de las características generales de los actores emergentes es su lógica dispersa. Frente a la institucionalidad y tiempos de gestión de las formas constituidas de la participación popular (Consejos Comunales y Comunas), los sindicatos y colectivos despliegan una variedad de relaciones con 
miembros del partido de gobierno, instituciones públicas y/o funcionarios, que les permiten rebasar con relativos rangos de efectividad, las trabas y dificultades propias de las "vías y procedimientos institucionales". En este sentido, las dinámicas expresadas por estos actores emergentes, no puede caracterizarse como una disputa franca y cruenta por modalidades y mecanismos de acceso a la renta petrolera. Por el contrario, las disputas y tensiones se despliegan alrededor de la legitimidad, el crédito y el reconocimiento derivado de una interlocución y gestión efectiva frente al Estado y la comunidad. En otras palabras, y desde lo observado en este punto del trabajo de campo, pareciera que no es el enriquecimiento ni el afán de acumular riquezas lo que activa el campo de disputas entre actores emergentes y actores consolidados, sino la búsqueda de legitimidad y reconocimiento (que luego podrán ser intercambiado como transacción no-simbólica en el mercado de los bienes políticos). Así, pese a la probabilidad de una "anomización” de los sectores más vulnerables a las contingencias económicas, la participación pareciera realizarse en la confluencia de intereses particulares y comunes que configuran un espacio de "lo común".

\section{CONCLUSIÓN EN CLAVE PROBLEMÁTICA}

Queda de manifiesto la complejidad de los procesos sociales en curso en la Venezuela chavista y la necesidad de una lectura que lejos de considerar estos ámbitos de acción colectiva como espacios "desocializados" (descompuestos socialmente, desorganizados y sin valores) que no alcanzarían la "verdadera" dimensión de la política, se sitúe en el campo de las disputas para caracterizar las nuevas prácticas que emergen en los territorios populares urbanos, sus efectos políticos más allá de las contingencias (acciones reivindicativas) y sus posibilidades. A su vez, queda también de manifiesto la necesidad de problematizar las lecturas alrededor del fenómeno del clientelismo, la cooptación y las condiciones de posibilidad de la autonomía. Hasta ahora, estas lecturas parecieran avalar la existencia de un "estigma originario" que privilegia el análisis de la efectividad 
de estas prácticas sólo en su relación con el Estado, restándole complejidad e importancia a las dinámicas constitutivas de los procesos políticos dentro de la comunidad.

Las dinámicas de la participación descritas abren la posibilidad de observar las dinámicas políticas en su dimensión estructural, es decir, a partir de las modalidades a través de las cuales los grupos se posicionan en el campo del poder y la magnitud con la que atraviesan y tensionan longitudinalmente a este último.

En primer lugar, vistas desde una lectura normativa y prescriptiva de la política, estas prácticas parecieran situarse en el ámbito de la acción defensiva y reivindicativa, lo que se traduciría en una limitación para el ejercicio de la política, que se expresa en la posición de los consejos comunales en los niveles de toma de decisiones dentro del modelo político. Es decir, los consejos comunales y las comunas son caracterizados, desde esta perspectiva, sólo como espacios de inclusión y resolución de problemas, y no como instancias de debate y reflexión política que le disputan poder, tanto a los partidos políticos como a las instancias de gobierno, por ejemplo.

Las preguntas que circundan el esfuerzo analítico arrojado aquí tornan alrededor de como se acepta o se efectúa la llegada de nuevos o reformulados grupos de actores (sindicato buscando apoyo de la Comuna para obtener mayor influencia en el territorio, llevando un discurso de reconciliación, unión y transformación radical; colectivo que aparenta ser un grupo sociodeportivo, sin uso de la fuerza coercitiva que caracteriza el "gremio" al cual se identifican) en un espacio controlado predominantemente por "clicas" políticas en base a la definición "antipolítica" (en el sentido de desconfianza hacia la política) de ellos (discurso del "poder popular", rechazo a las instituciones, imbricación partido/organización comunal no discutida o puesta en tela de juicio). Las comunas, en este contexto, sirven de vaso comunicante a luchas latentes en el campo político-institucional $^{20} \sin$ hegemonizar a ningún grupo en particular - por ahora

\footnotetext{
${ }^{20}$ Sería interesante buscar analogía de situación con la emergencia piquetera y el desplazamiento de la influencia de los punteros justicialistas en Argentina, o en la relación entre la FEJUVE y el MAS en Bolivia aunque parte de un movimiento social más estructurado alrededor de luchas obreras y campesinas y confluyendo en la penetración en sectores reacios a la política y representante de un subproletariado desagregado.
} 
- como ha podido suceder en el caso brasileño en los noventa con una predominancia de lo ilícito en las políticas populares a través de las milicias, de los narcotraficantes y las asociaciones de moradores.

En segundo lugar, si la participación política y social, más allá del voto o de formas de voluntariado local, da mucha enseñanza sobre la calidad democrática cuyo nivel se mide función de esos dos indicadores, el hecho de que haya integración o acercamiento de nuevos actores es de primera importancia. En tanto cómo el poder popular puede expresar un reflejo, una voluntad colectiva variopinta, diversa, su mayor fuerza radica en su capacidad en establecer nuevos campos de diálogos y confrontación de ideas e intereses. Estos espacios de fricciones de lógicas e intereses divergentes aunque pertenecientes a un amplio espectro popular de prácticas políticas, pueden remitir a los espacios insurgentes a los cuales se refiere Holston (2007) que sustituyen o conquistan espacios dejados por el Estado (en término de regulación social, sea el caso de la violencia o del trabajo). O si bien, por el contrario, remite a la naturaleza dual del Estado, tanto campo en disputa y objeto de botín característico de un patrimonialismo "salvaje" de los cuales los grupos mencionados serían un reflejo en el terreno local.

En tercer lugar, los procesos citados problematizan el ámbito de la distinción entre lo público y lo privado a través de mecanismos de disputas por la apropiación de las instancias que constituyen el campo político de los territorios populares urbanos, principalmente, en lo relacionado a las políticas de financiamiento, sus objetivos y sus marcos de gestión. Sin embargo, lejos de plantear -en términos absolutos- el devenir de estas prácticas como una consecuencia de procesos "interventores" por parte del Estado, habría que pensar si -efectivamente- son expresiones de la fragilidad, precariedad y vulnerabilidad del lazo social en un contexto tendiente al individualismo, atravesado por los riesgos del clientelismo y la informalidad en el mundo del trabajo.

En cuarto lugar, el proceso en el que se transversalizan las trayectorias, en las disputas y tensiones dentro del campo de cooperación, alianzas y/o conflictos en el marco de las Comunas, el epi- 
centro de las sociabilidades tiende a articularse en torno a dos ejes básicos: la violencia y el (des)crédito. Así, es posible rastrear dentro de cada uno de los ejes básicos citados, en primer lugar, las disputas alrededor de la definición de un "nosotros" comunitario que lejos de ser estable se construye a partir de estrategias de reconocimiento mutuo (y de una comunidad imaginada, de lo nacional y lo popular); que mientras se integran algunos actores se condenan y excluyen a otros. Finalmente, están las dimensiones que constituyen los campos de interlocución, interpelación e intermediación entre ese "nosotros" como comunidad y el Estado.

Partiendo de estas reflexiones y resultados provisionales, podríamos analizar la importancia del capital político de cada uno de los dos grupos de actores, o sus mutaciones, para centrarnos en un incipiente y creciente capital militante que se caracteriza por el don de sí, el compromiso consciente y una acción circunscrita y eficaz. Más precisamente, “el capital militante designa, más allá de la diversidad de las formas de compromiso, un saber-hacer adquirido, en particular gracias a propiedades sociales que permiten jugar, con mayor o menor éxito, en un espacio que está lejos de estar unificado » (Poupeau 2007:44). Esa politización sui generis, o cultura política participativa, sirve de base para emprender el análisis de las formas diversas de poder popular, ancladas en prácticas disruptivas (o incluso transgresivas) y relacionadas con la superposición del campo económico-laboral y campo político ${ }^{21}$. El despliegue de esta modalidad de análisis, centrada en las imbricaciones más que en las tensiones, permitiría avanzar - progresivamente- en la definición de un marco que defina y caracterice el trazo de estos nuevos recorridos, a contrapelo de las lecturas de carácter populista y sustancialista que idealizan las prácticas del poder popular, sin apreciar las mutaciones del campo popular tensionado, a la vez que parcelado y reconfigurado, por lógicas de acumulación políticas y económicas.

${ }^{21} \mathrm{Al}$ estilo de los trabajos emprendidos por Vera Telles (2014) sobre la gestión disputada del orden. 


\section{REFERENCIAS}

ADDOR. F. Teoria democrática e poder popular na América Latina. Contribuições a partir das experiências de Cotacachi/Equador e Torres/Venezuela. Tese (Doutorado em Planejamento Urbano e Regional), Universidade Federal do Rio de Janeiro, 2012.

ALVAREZ, S.; DAGNINO, E.; ESCOBAR, A. (Eds.). Culture of politics. Politics of Culture: Revisioning Latin American Social Movements. Boulder: Westview Press, 1998.

ANTILLANO, A. La lucha por el reconocimiento y la inclusión en los barrios populares: la experiencia de los Comités de Tierras Urbanas. Revista Venezolana de Economía y Ciencias Sociales. Vol. 11. nº. 3, pp.205-218, 2005.

AUYERO, J. La política de los pobres. Las prácticas clientelistas del peronismo. Buenos Aires: Manantial, 2001.

AUYERO, J. La zona gris. Violencia colectiva y política partidaria en la Argentina contemporánea. Buenos Aires: Siglo XXI Editores, 2007.

BLONDIAUX, L. Le nouvel esprit de la démocratie. París: Le Seuil, 2008.

CICCARIELO-MAHER, G. We Created Chávez: A People's History of the Venezuelan Revolution. Durham/London: Duke University Press, 2013.

DENIS, R. Los fabricantes de la rebelión (Movimiento popular, chavismo y sociedad en los años 90). Caracas: Editorial conjunta Primera Línea y Nuevo Sur, 2000.

GARCÍA-GUADILLA, M. "La praxis de los consejos comunales: ¿poder popular o instancia neoclientelar?" In: Revista Venezolana de Economía y Ciencias Sociales, Vol. 14, nº1, pp.125-151, 2008.

GEERTZ, C. La interpretación de la cultura. Barcelona: Gedisa, 1992.

HANSON, R. Looking at the Collaboration between the Police and the Collectives, extraído el 17 de mayo del 2014, http://anthropoliteia. net/2014/05/13/views-of-venezuelan-protests-pt-4-looking-at-thecollaboration-between-the-police-and-the-collectives/, 2014.

HARNECKER, M. Venezuela. Alcaldía de Caroní. Gobernar: tarea de todos. Caracas: Editorial Fundarte, 1994.

HOLSTON, J. Insurgent Citizenship : Disjunctions of Democracy and 
Modernity in Brazil, Princeton: Princeton University Press, 2007.

HURTADO, S. Dinámicas comunales y procesos de integración social: las organizaciones populares. Caracas: Fondo Editorial Tropykos, 1991.

IVANOVIC, M. La democracia en tiempos de revolución, El caso venezolano. In: GAUDICHAUD, F. (Coord.). Emancipaciones en América latina. Quito: IAEN, 2013, pp. 61-72.

LACLAU, E.; MOUFFE, C. Hégémonie et stratégie socialiste: vers une politique démocratique radicale. Besançon: Les Solitaires intempestifs, 2009.

LANDER, E. Neoliberalismo, sociedad civily democracia. Caracas:Consejo de Desarrollo Científico y Humanístico de la Universidad Central de Venezuela, 2006.

LÓPEZ MAYA, M. Innovaciones participativas y poder popular en Venezuela. In: PACHANO, S. Temas actuales y tendencias en la ciencia política. Quito: Flacso, 2008, pp.197-205.

MARX, K. La guerre civile en France. Adresse du Conseil général de l'Association internationale des travailleurs. In: BENSAID, D. Inventer l'inconnu. Textes et correspondances autour de la Commune. Paris: La Fabrique, 2008, pp. 50-61.

MATO, D. Políticas de ciudadanía y sociedad civil en tiempos de globalización. In: MATO, D. (Coord.). Politicas de Ciudadanía y Sociedad Civil en tiempos de globalización. Caracas: Facultad de Ciencias Económicas y Sociales, Universidad Central de Venezuela, 2004.

MERKLEN, D. Pobres ciudadanos. Las clases populares en la era democrática (Argentina, 1983-2003). Buenos Aires: Gorla, 2005.

OSSANA, J. Punteros, malandras y porongas. Ocupación de tierras y usos politicos de la pobreza. Buenos Aires: Siglo XXI, 2014.

POGREBINSCHI, T. El giro pragmático de la democracia en América Latina. Buenos Aires: Nueva Sociedad, junio 2013.

POUPEAU, F. Dominación y movilizaciones. Estudio sociológico sobre el capital militante y escolar, Córdoba, Ferreyra Editor,pp. 2007.

REY, J. C. La democracia venezolana y la crisis del sistema populista de conciliación. In: Revista de Estudios Políticos, nº74, pp.533-578, 1991. 
REY TORRES, E. Territorialidad y formas de sociabilidad : aprendizajes de la experiencia de los Comités de Tierras Urbanas. Tesis de Grado para optar al título de sociólogo, Escuela de Sociología, Facultad de Ciencias Económicas y Sociales (FaCES), Universidad Central de Venezuela, 2011.

ROSA BONHEUR. Comment étudier les classes populaires aujourd'hui? Une démarche d'ethnographie comparée, Espaces et sociétés, Vol. 1, n¹56-157, pp.125-141, 2014.

SALAMANCA, L. Movimiento vecinal y democracia. La sociedad contra el Estado- partido. Sic. Caracas: Centro Gumilla. Vol. 50, nº.500, diciembre, pp.522-525, 1987.

TELLES, V. Gestion de la violence ou gestion (disputée) de l'ordre? Interrogations à partir d'une étude sur le marché de la drogue à São Paulo. In: L'ordinaire des Amériques. 126, http://orda.revues. org/1120, extraido el 12/02/2014, 2014.

TRIGO, P. Organización popular e identidad barrial en Caracas, Nueva Sociedad, n.136, pp. 96-111, 1995.

VARGAS, A. La praxis de Consejos Comunales y la Visión de las Élites sobre la Democracia Participativa. CIES- Working Paper, n 95 , 2010 .

Recebido: 31/03/2015.

Aprovado: 20/07/2015. 